\title{
Ultrasound-guided breast biopsy of ultrasound occult lesions using multimodality image co-registration
}

\author{
Anton Nikolaev $^{1 *}$, Hendrik H.G. Hansen ${ }^{1}$, Leon de Jong ${ }^{1}$, Eleonora Tagliabue ${ }^{2}$, Bogdan \\ Maris $^{2}$, Vincent Groenhuis ${ }^{3}$, Françoise Siepel ${ }^{3}$, Marco Caballo ${ }^{1}$, Ioannis Sechopoulos ${ }^{1,4}$ and \\ Chris L. de Korte ${ }^{1,3}$ \\ ${ }^{1}$ Department of Radiology and Nuclear Medicine, Radboud University Medical Center, \\ Nijmegen, The Netherlands, ${ }^{2}$ Department of Computer Science, Università degli Studi di \\ Verona, Verona, Italy, ${ }^{3}$ University of Twente, Enschede, The Netherlands ${ }^{4}$ Dutch Expert \\ Center for Screening (LRCB), Nijmegen, The Netherlands
}

*anton.nikolaev@radboudumc.nl

\begin{abstract}
Fusion-based ultrasound (US)-guided biopsy in breast is challenging due to the high deformability of the tissue combined with the fact that the breast is usually differently deformed in CT, MR, and US acquisition which makes registration difficult.

With this phantom study, we demonstrate the feasibility of a fusion-based ultrasound-guided method for breast biopsy. 3D US and 3D CT data were acquired using dedicated imaging setups of a breast phantom freely hanging in prone position with lesions. The 3D breast CT set up was provided by Koning (Koning Corp., West Henrietta, NY). For US imaging, a dedicated breast scanning set up was developed consisting of a cone-shaped revolving water tank with a 152-mmsized US transducer mounted in its wall and an aperture for needle insertion. With this setup, volumetric breast US data $\left(0.5 \times 0.5 \times 0.5 \mathrm{~mm}^{3}\right.$ voxel size $)$ can be collected and reconstructed within 2 minutes. The position of the lesion as detected with breast CT was localized in the US data by rigid registration. After lesion localization, the tank rotates the transducer until the lesion is in the US plane. Since the lesion was visible on ultrasound, the performance of the registration was validated.

To facilitate guided biopsy, the lesion motion, induced by needle insertion, is estimated using cross-correlation-based speckle tracking and the tracked lesion visualized in the US image at an update frequency of $10 \mathrm{~Hz}$.

Thus, in conclusion a fusion-based ultrasound-guided method was introduced which enables ultrasound-guided biopsy in breast that is applicable also for ultrasound occult lesions.
\end{abstract}

Keywords: ultrasound guided biopsy, multimodality image fusion, 3D breast ultrasound, lesion displacement tracking

\section{INTRODUCTION}

Cost effectiveness, high usability and real-time performance make ultrasound the first imaging modality to use for biopsy guidance. Unfortunately, ultrasound-guided biopsy is no longer applicable if the lesion is US occult. In this case, an alternative solution for the patient is more expensive and time consuming MRI-guided biopsy. It becomes even more complicated for patients with contraindications to MRI for instance for patients with claustrophobia or metal implants. 


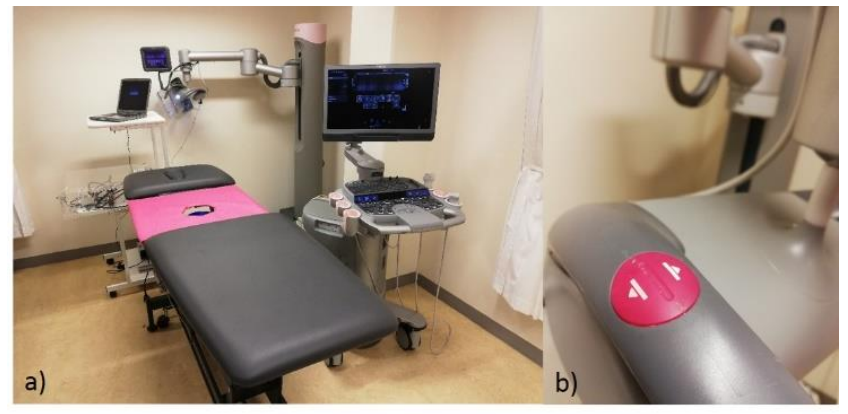

Fig. 1 - Automated breast ultrasound scanning and biopsy system (a) and button to initiate the data acquisition (b)

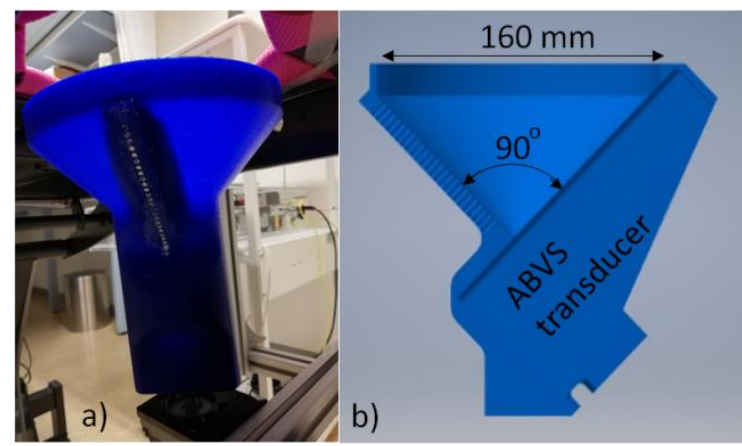

Fig. 2 - Cone shaped container (a) and its schematic (b)

A solution for this can be an ultrasound-guided biopsy based on image fusion where the lesion in the US image is localized based on a co -registration with images obtained from other imaging modalities. For instance, MRI

- US guided prostate biopsy is already widely used in clinic [1]. Unfortunately, it is challenging to apply this technique in breast due to the large deformation introduced.

This possible solution based on MRI - US image fusion, has already been proposed for breast tissue in [2]. In this phantom study the phantom was fixed between two plates similar to those used MRI-guided biopsy. The phantom was repositioned using a detachable table. Because of the fixation, the phantom had the same shape during both MRI and US scanning which facilitated rigid image co-registration and in that way the problem related to deformations of the breast were circumvented.

In this work we demonstrate a different approach of ultrasound-guided breast biopsy of lesions applicable also to lesions that have low contrast with surrounding tissue or are not even visible with ultrasound. As a screening modality we used dedicated breast CT [3]. The primary challenges of the project are to enable registration between breast CT and ultrasound volumetric data without breast fixation to increase patient comfort. The 3D ultrasound imaging was performed with a dedicated setup where the breast was prone positioned during the scanning and not deformed.

Additionally, we propose the use of a speckle tracking algorithm [4] to improve targeting of the lesion when it is displaced from the registered position upon insertion of the biopsy needle [5].

The method was validated using phantom experiments.

\section{METHODS}

\section{System description and the workflow}

The automated breast ultrasound scanning and biopsy (ABUS - BS) system is depicted in Fig. 1. The system incorporates an examination table, a Siemens ACUSON S2000 system (Siemens Medical Solutions, Mountain View, CA, USA) and a cone-shaped PLA breast container with a Siemens 14L5BV transducer (Siemens Medical Solutions, Mountain View, CA, USA) integrated underneath the examination table Fig 2a. An array of circular apertures has been created in the side of the cone opposite to the transducer to facilitate the introduction of biopsy needles. The cone can make a $360^{\circ}$ revolution around the breast. 

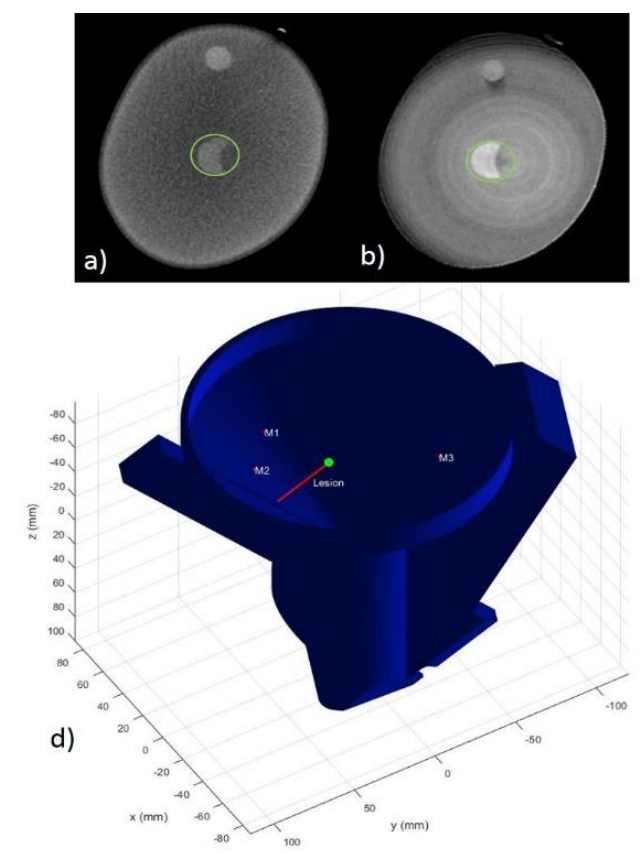

Fig. 3 - Example of image co-registration (a) and lesion localization in a cone-related coordinate system (b) using the custom SW

The target patient population for the ABUS - BS system is women who developed suspicious lesions that are hardly visible or occult in US. First, a 3D dedicated breast CT (Koning Corp., West Henrietta, NY) of the patient's breast was acquired (this could also be a breast MRI). Next, a 3D US acquisition of the breast is performed.

For the US procedure, the patient lays down on the examination table (Fig. 1a) in prone position placing her breast inside of the breast container as depicted in Fig 1. When the patient is in the right and comfortable position, the sonographer can start the procedure.

The procedure can be separated in US volumetric scanning and biopsy. To start breast volumetric scanning, the radiologist only needs to press the button on the ABVS system (Fig. 1b) to initiate the cone revolution. Next, the radiologist should upload the acquired 3D DICOM data into the custom SW. Based on the volumetric CT and US image fusion (Fig. 3a), the SW outputs the navigation parameters: the cone rotational angle, the number of the most optimal aperture for insertion and the depth to which the needle should be inserted (Fig. 3b). The cone then rotates to this specific rotational angle, at which the lesion can easily be targeted. Next, the radiologist inserts the needle through the indicated aperture, brings the needle to the target, while getting real-time imaging feedback from the ultrasound probe which is in the same 2D plane. Finally the biopsy sample is taken.

\section{System Design}

An Acuson S2000 system is used for the US imaging and the data acquisition with the 152-mm-wide (768 elements) ABVS transducer. B-mode images are $60 \mathrm{~mm}$ in the axial direction. The transducer is mounted into a cone-shaped container. Due to the fixation, part of the transducer is blocked, leaving only $114.3 \mathrm{~mm}$ available for imaging (Fig. 2b). The opening angle of the cone is $90^{\circ}$ and the diameter of the cone's base is $160 \mathrm{~mm}$. The container's design allows breasts of cup size A and B to hang free inside of the container and to be naturally aligned by the gravity alike the breast CT scanning. The cone is filled with water as an acoustic coupling medium.

An array of through holes $(2.5 \mathrm{~mm}$ diameter and $1.5 \mathrm{~mm}$ gap in between apertures) on the opposite of the transducer side facilitates the biopsy. The holes provide needle steering in parallel to the transducer surface. This design prevents any contact between the transducer and the needle to avoid damaging.

The container is actuated by the closed loop stepper motor ARM69AC (Oriental Motor, Torrance, USA) that revolves $360^{\circ}$ in 60 seconds. During the revolution 316 US frames $\left(1.13^{\circ}\right.$ between acquired US frames) are acquired by the US system. The total duration of the US image acquisition is $60 \mathrm{sec}$. The motor's revolution is triggered with encoding pulses from the stepper motor of the ABVS robotic arm.

Acquired volumetric DICOM data are then uploaded into the custom SW and volumetric image reconstruction and registration with the breast CT image is performed, followed by the biopsy navigation. 


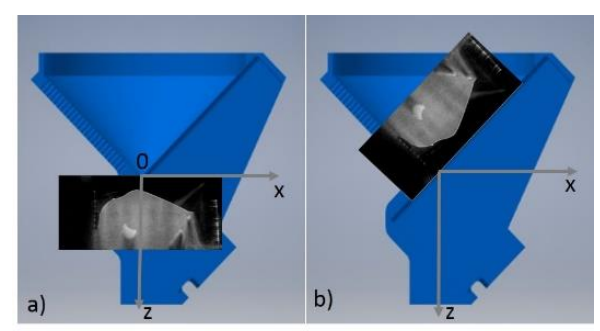

Fig. 4 - US frame in cone-related coordinate system before (a) and after (b) transformation

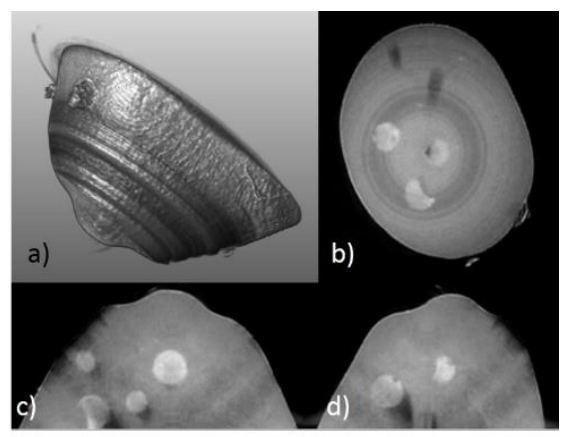

Fig. 5 - 3D reconstructed ultrasound volume (a) of the breast phantom and its cross-sections in coronal (b), sagittal (c) and transversal (d) planes

The 3D volume is reconstructed from the cloud of points by assigning to each voxel the median value of pixels[6], belonging to the voxel. The holes remaining after the reconstruction are filled with linear interpolation. The cloud of points is formed by transforming each pixel of each initial B-mode image $\mathrm{p}^{0}$ into a corresponding location of the cone's spatial coordinate system using linear transformation 1.

$$
\begin{aligned}
& \mathrm{p}^{\mathrm{n}}=\mathrm{H}_{\mathrm{T}}^{\mathrm{C}} \mathrm{p}^{0}, \\
& \mathrm{H}_{\mathrm{T}}^{\mathrm{C}}=\operatorname{Rz}(1.14 * \mathrm{n}) * \mathrm{Ry}\left(180+\frac{\alpha}{2}\right) * \mathrm{~T}, \\
& \mathrm{~T}=\left[\begin{array}{cccc}
1 & 0 & 0 & \mathrm{w} / 2 \\
0 & 1 & 0 & 0 \\
0 & 0 & 1 & 0 \\
0 & 0 & 0 & 1
\end{array}\right]
\end{aligned}
$$

Where $\mathrm{p}^{0}$ represents the pixels belonging to the US image, $\mathrm{H}_{\mathrm{T}}^{\mathrm{C}}$ is the transformation matrix from the transducer's coordinates to the cone's coordinates, $\mathrm{Rz}$ and $\mathrm{Ry}$ are rotation matrixes representing rotation around $\mathrm{z}$ and $\mathrm{y}$ axis respectively, $w$ is the width of the transducer, $n$ is the number of acquired frames. A resulting 3D US volume of the phantom is depicted in Fig. 5.

Next, a rigid marker based registration is done to localize the lesion in the cone originated coordinate system. The marker segmentation was done manually by the sonographer on both 3DUS and CT images. On the screening image radiologist also needs to segment the lesions to be biopsied. Next, the CT volume is registered to US image in cone originated coordinate system using gradient decent algorithm. After the lesion localization, SW outputs the angle $\beta$ ( equation 5) to rotate the cone until the center of the lesion belongs the transducers plane and number of biopsy aperture to use for the intervention, and the distance from the aperture to the center of the lesion.

The aperture to use for the biopsy $\mathrm{N}_{\text {aperture }}$ is the closest aperture to the projection $\mathrm{B}\left(\mathrm{B}_{\mathrm{x}}, \mathrm{B}_{\mathrm{y}}, \mathrm{B}_{\mathrm{z}}\right)$ of the center of the lesion $\mathrm{L}\left(\mathrm{L}_{\mathrm{x}}, \mathrm{L}_{\mathrm{y}}, \mathrm{L}_{\mathrm{z}}\right)$ on the opposite the transducer cone forming line $\mathrm{v}^{\mathrm{b}}$. In case of the straight cone angle $\alpha$, the aperture can be found from the equations 5 and 6 . 


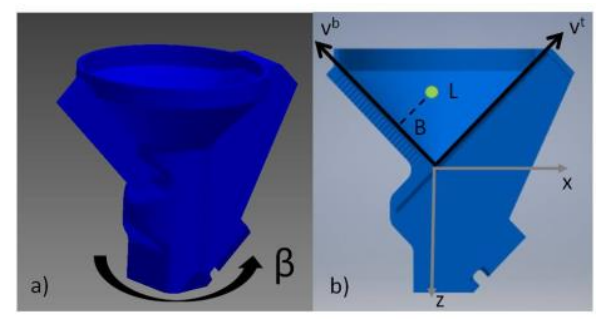

Fig. 6 - Cone navigation parameters. Picture a depicts direction of the rotation and picture (b) depicts estimation of the biopsy aperture

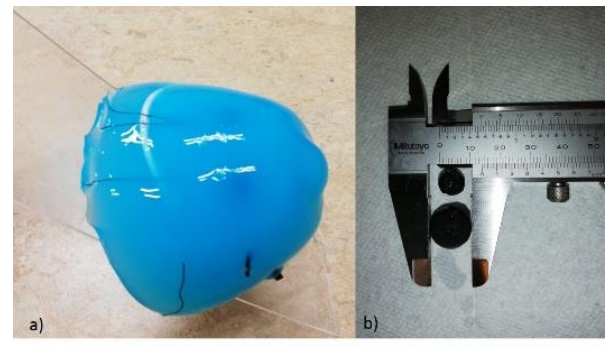

Fig. 7 - PVA breast phantom (a) and lesions inside (b)

$$
\begin{aligned}
& \beta=\operatorname{atan}\left(\frac{L_{y}}{L_{x}}\right), \\
& N_{\text {aperture }}=\left[\frac{\sqrt{B_{x}^{2}+B_{z}^{2}}}{\Delta}\right], \\
& {\left[\begin{array}{cc}
v_{x}^{b} & v_{z}^{b} \\
v_{x}^{t} & v_{z}^{t}
\end{array}\right]\left[\begin{array}{l}
B_{x} \\
B_{z}
\end{array}\right]=\left[\begin{array}{c}
v_{x}^{b} L_{x}+v_{z}^{b} L_{z} \\
0
\end{array}\right],}
\end{aligned}
$$

where $\mathrm{v}^{\mathrm{t}}$ is a vector collinear to the transducers lateral direction, $\mathrm{v}^{\mathrm{b}}-$ vector collinear to opposite the transducer cone forming line, $\Delta$ - distance between centers of two biopsy apertures $(3.25 \mathrm{~mm})$.

\section{Phantom validation}

The procedure was validated on a breast phantom with 8 lesions: 4 lesions of $7 \mathrm{~mm}$ in diameter and 4 lesions of $13 \mathrm{~mm}$ in diameter. The phantom was fabricated by dissolving poly vinyl alcohol (PVA) (Acros organics, Geel, Belgium) in antifreeze liquid and adding 1\% of Silica gel 60 (Merck KGaA, Darmstadt, Germany)to create a homogenous speckle pattern.

The lesions were fabricated first by using $10 \%$ of PVA, $84 \%$ of antifreeze liquid and $5 \%$ of black paint. The dissolved PVA was poured into spherical mold and then frozen once. Next, the lesions were fixed inside of the breast mold.

The phantom was fabricated by using 5\% PVA and 94\% of antifreeze liquid. The $22^{\circ}$ solution was poured into the breast mold and frozen.

The lesions did not have a perfect spherical shape because of the groove formed during the manufacturing. Both lesions and phantoms contain the same concentration of silicon particles for the homogeneous speckle pattern.

The phantom was biopsied 5 times with a 16G ACECUT biopsy needle (TSK Laboratory, Japan) and the number of successfully biopsied lesion was assessed by visually assessing the presence of staining.

\section{Breast tissue deformation}

To track the lesion displacement induced by needle insertion or other external motions, we used cross-correlationbased tissue motion estimation [7] with a template of $5.6 \mathrm{~mm}$ x $4.5 \mathrm{~mm}$ and a search kernel of $112 \times 102 \mathrm{~mm}$. A region of interest (ROI) around the lesion to be tracked was marked automatically after the image fusion step. On each iteration, displacement of each pixel within the ROI along the axial and lateral directions was estimated. The average displacement of all pixels within the ROI was used to update the center position of the lesion from a previous ultrasound frame to the current. In this way the tracking the ROI means monitoring of the lesions 


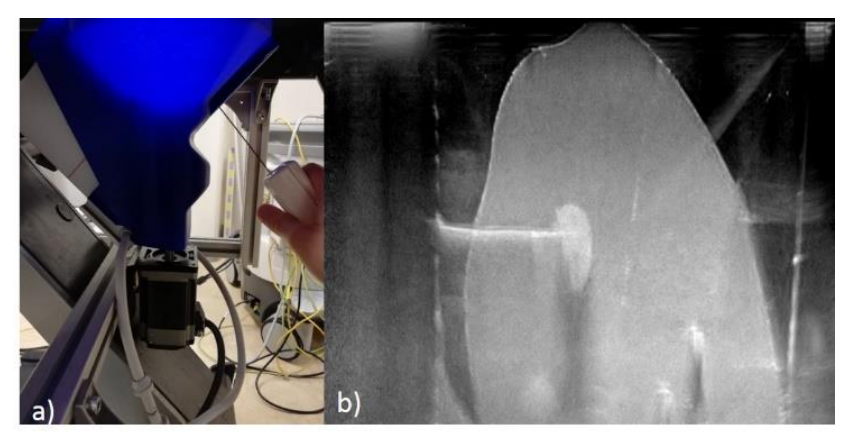

Fig. 8 - Biopsy using the cone-shaped container (a) and B-mode image during the intervention (b).

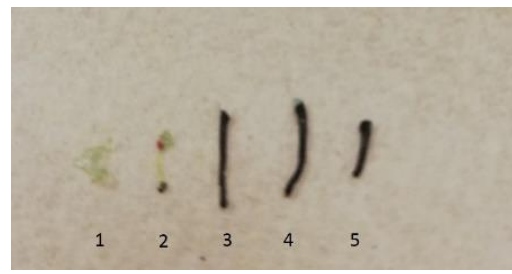

Fig. 9 - Result of the biopsies taken from the PVA phantom with color-stained lesions
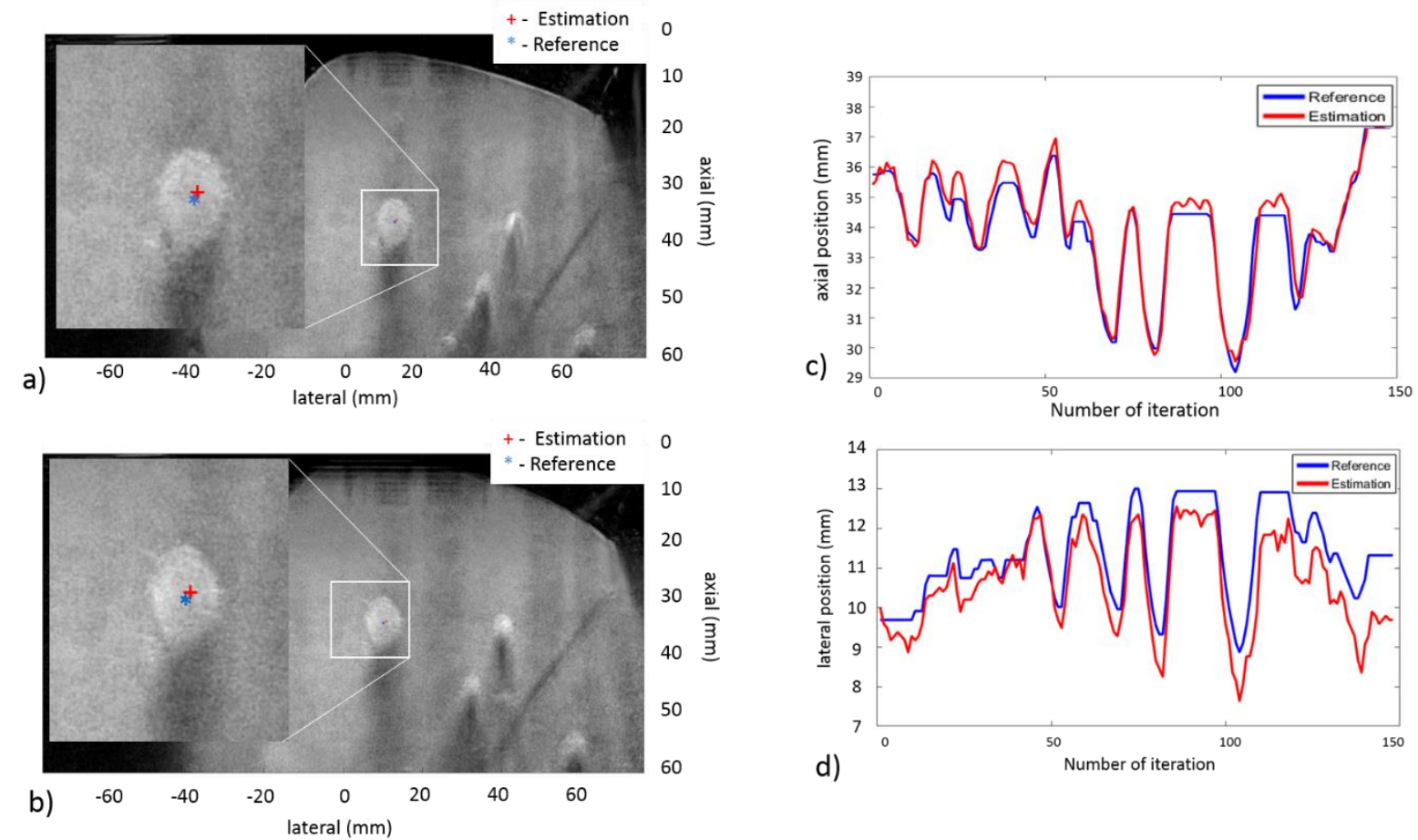

Fig. 10 - Displacement before (a) and after (b) the deformation and lesion trajectories along axial (c) and longitudinal (b) directions. Blue color depicts a real ground truth displacement and red color estimated displacement.

displacement. To improve the tracking accuracy, only displacement vectors collinear to the mean displacement vector were taken into consideration for ROI displacement estimation. The lesion tracking was validated experimentally. The deformation of the breast was introduced by pushing the breast manually against the inside of the container.

The center of the tracked lesion was manually segmented in the recorded B-mode images. The median and standard deviation of the distance between the manually segmented center and the center of the lesion estimated using displacement tracking was calculated to quantify the accuracy and precision of the lesion tracking. 

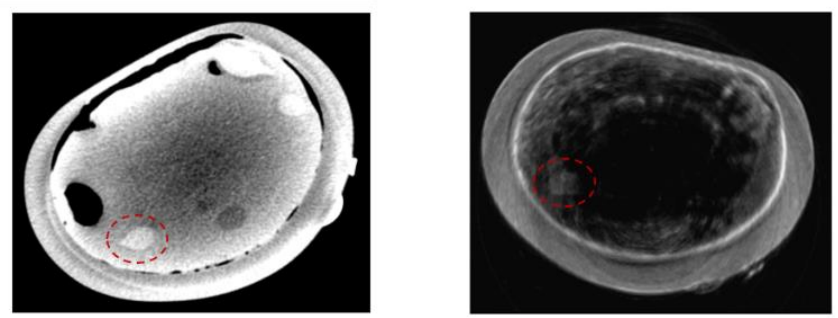

Fig. 11 - Lesion registration in CIRS (CIRS M73, InMed, Seven Hills) phantom where the lesion is now clearly visible in ultrasound.

\section{RESULTS}

Biopsy

The time between placement of the phantom on the table and biopsy is 3 minutes including 1 minute of scanning and 2 minutes for the 3DUS image reconstruction at a resolution of $0.5 \times 0.5 \times 0.5 \mathrm{~mm}^{3}$. The phantom was biopsied 5 times: 3 times in small lesions and 2 times in large lesions. From the obtained samples (Fig. 9) we can see that 4 out of 5 samples contained staining of the lesions, thus indicating successful biopsy. The sample 1 was not stained and lesion 2 was stained only partially. Sample number 5 was a successful example of a small lesion biopsy. Samples 3 and 4 corresponded to successfully biopsied large lesions.

\section{Displacement}

The displacement estimation took approximately $0.1 \mathrm{~s}$ to calculate. Thus, lesion tracking appears to be possible at $10 \mathrm{~Hz}$. The results of the displacement tracking are shown in Fig. 10. The maximum lesion displacement observed was $9 \mathrm{~mm}$. During the motion the mean tracking error was $0.9 \mathrm{~mm}$ with a standard deviation of $0.4 \mathrm{~mm}$. Thus, the cross-correlation based method was able to track the actual lesion displacement.

The result of the displacement tracking is shown on the Fig. 10. The maximum lesion displacement observed was $9 \mathrm{~mm}$. During the motion the mean tracking error was $0.9 \mathrm{~mm}$ with standard deviation of $0.4 \mathrm{~mm}$.

\section{DISCUSSION}

This study demonstrates the feasibility of the approach with the novel cone-based system. In future studies the in vivo performance of the method will have to be examined. Furthermore, the reproducibility of the method will have to be investigated by having various people perform the biopsy several times. In this study we used the US/breast CT compatible phantom to demonstrate the feasibility of the method. In future work, the marker-based registration can be substituted by surface registration to make it possible for the patient to do the screening and the biopsy on different days.

The proposed procedure is simple, doesn't require any special training and can be performed without looking at the screen. The procedure can easily be applied also for lesions that are hardly visible on US as in Fig. 11.

However, we were not successful in all biopsies: one lesion was not biopsied. The reason for this is that during the biopsy the needle was out of the transducers B-plane. Each biopsy aperture is $2.5 \mathrm{~mm}$ when the needle is 1.3 $\mathrm{mm}$. This mismatch caused a deviation of the needle from the initial trajectory and consequent mistargeting. The same situation happened while targeting the second lesion. Due to the deviation was not so large and thanks to the prior information about the lesion remoteness from the cones wall, it was still possible to biopsy the lesion. In 3 other cases, the needle was visible in ultrasound image and the needle was following preplanned path. The recommended value for the aperture holes would be $1.3 \mathrm{~mm}$ diameter. This will decrease the chance of mistargeting.

A second possible reason that can result in a failed biopsy in vivo is lesion motion induced by the needle insertion. Although in the described experiment the needle insertion did not cause the lesion to move, this has been described before for in vivo measurements. Nevertheless, we demonstrated that the cross-correlation-based method can provide an accurate lesion tracking giving the radiologist the opportunity to correct the in plane needle position during biopsy. 


\section{CONCLUSION}

The project introduces a method for image-fusion-based ultrasound-guided breast biopsy. It is demonstrated that ultrasound-guided biopsy of low contrast or ultrasound occult lesions is possible by using image fusion and tissue displacement estimation. Potentially, this method can be clinically used and be an alternative to MRI-guided biopsy.

The introduced cone-based acquisition method to facilitate 3D US registration to 3D dedicated breast CT has not been described before. Until now, published breast scanning setups were designed for breast screening and required contact between the breast surface and US transducer, which resulted in breast deformation and did not allow rigid registration with other images from other modalities.

\section{REFERENCES}

1. Marks L., Y.S., Natarajan S., MRI-ultrasound fusion for guidance of targeted prostate biopsy. NIH, 2013. 23(1).

2. Piron, C.A., et al., A hybrid breast biopsy system combining ultrasound and MRI. IEEE Trans Med Imaging, 2003. 22(9): p. 1100-10.

3. Boone, J.M., et al., Dedicated breast CT: radiation dose and image quality evaluation. Radiology, 2001. 221(3): p. 657-67.

4. Lopata, R.G., et al., Performance evaluation of methods for two-dimensional displacement and strain estimation using ultrasound radio frequency data. Ultrasound Med Biol, 2009. 35(5): p. 796-812.

5. Jorn op den Buijs, H.H.G.H., Richard G. P. Lopata, Chris L. de Korte, Sarthak Misra, Predicting Target Displacements Using Ultrasound Elastography and Finite Element Modeling. IEEE transactions on biomedical engeneering, 2011. 58(11): p. 13.

6. Solberg, O.V., et al., 3D ultrasound reconstruction algorithms from analog and digital data. Ultrasonics, 2011. 51(4): p. 405-19.

7. Lopata, R.G., et al., Comparison of one-dimensional and two-dimensional least-squares strain estimators for phased array displacement data. Ultrason Imaging, 2009. 31(1): p. 1-16. 